\title{
CURRENT STATUS OF PROJECTIVE TEST THEORIES AND PROJECTIVE TECHNIQUES
}

\author{
GORDON F. DERNER
}

Adelphi College, L.I., New York

In order to sharpen the present review of the current status of projective test theory and projective techniques, it is focused on the more recent developments in the United States over the past several years. To begin the review, however, we first need to look back to the important beginnings of projective techniques in the brilliant contributions of Herman Rorschach.

Dr. Rorschach, in attempting to sharpen procedures for the diagnosis of schizophrenia, experimented with ink blots. In his investigations he made the brilliant deduction that a most cogent way of understanding a person was to evaluate the structure of the subject's responses rather than focus on their content. Further he proposed that analysis of the interaction between movement and color responses would afford an intimate glimpse into the way the subject viewed the world and himself. Rorschach's interest was essentially clinical, however, even though he made sterling contributions to personality and perception theory.

In the earlier use of projective techniques, while they were yet to be so named by Lawrence $\mathrm{K}$. Frank, the primary emphasis was on their clinical usefulness. The diagnosis of psychopathology tended to be the important focus. Gradually an important shift has occurred. In a 1957 review prepared by Dr. Bertram R. Forer, the Executive Editor of the Journal of Projective Techniques, he noted this shift in emphasis on the papers contributed for publication. The shift can best be described as an increased use of projective techniques as research tools which are used to study broader psychological theory.

The basic theory of projective techniques, that dynamic factors influence our perception, continues to be a central concept in projective test theory. In some unclear fashion our past makes us interpret amorphous, semi-amorphous, or even quite clear material, frequently but not always, in a highly personal way. Our needs, our drives, our value systems, our ego defense systems, our self-systems all interrelate to determine these perceptions. They establish a perceptual set. In using projective techniques the psychologist obtains a measure of the subject's perceptual set and then works backwards on the basis of inference to what appear to be the subject's needs or drives. The subject's perceptions are selective so a measure of his ego and ego defenses can be interfered. The perceptions also reflect the selective inattention which serves as a defense. Perceptions can reflect the subject's persistent tensions. The perceptual set and the subject's responses to the stimulus material reflect the inner or intrapsychic state. The psychodynamic picture of the subject can be obtained but projective test theory has moved far beyond the limited concern with only the interaction between the intrapsychic state and the stimulus. The perceptual set is influenced by the stimulus material but it is also influenced by the examiner. The subject's responses can be altered as a function of the personality and demeanor of the examiner as well as his instructions to the subject. The alterations in responses which can be so brought 
about make it possible for projective techniques to serve as a measure of the dependent variable in research design.

Projective test theory now fully alert to the influence of the interpersonal and situational variables can study the intrapsychic significance of responses in a new light. We now know that it is generally futile to attempt to discover a simple one to one relationship between a fantasy production and an item of overt behavior. A direct interpretation of fantasy productions as equal to actual behavior has led to a not infrequent cynical attitude about the usefulness of projective techniques. When used in such a limited fashion projective techniques have contributed little to personality theory.

Personality evaluation and projective technique theory, however, has grown beyond the naive expectations of the simple equating of fantasy with predictions of observable behavior. With the broader perspective the usefulness of projective techniques in personality research has increased. To give an example of the more sophisticated understanding of the interpretation of the perceptual response, let us look at the trait of aggression. In a fantasy production the aggressive impulse may be manifest quite openly but a careful evaluation of the perceptions reported in the test protocol may show that an equally potent control system serves to contain the impulse. In overt behavior then the subject would have thoughts of aggressive acts but because of the effective control system, he would not act on the aggressive impulses. A balance between the impulse system and the control systen could mean that provocation would need to be very high for the impulse to be manifest in overt behavior. If the control system is high, provocation would need to be very high to have the aggression brought forth. Another model would exist when the impulse system is low. In this instance the control system could be quite weak and no overt aggressive acts would be seen. Further, the fantasy may be compensatory so that the impulse would be expressed in fantasy only and would not be expressed in actuality.

In some quite intriguing research by my Adelphi College colleague Dr. Gerald S. Lesser, the interplay of social sanction and the fantasy expression or overt expression of aggression was clearly shown. Lesser found that children who were raised in sub-cultures which permitted the forthright display of aggression did have aggressive themes in their projective techniques protocols and would be aggressive to overt behavior. Those subjects who were members of sub-cultures which were unaccepting of overt aggression and who showed high impulse toward aggression also showed high control in their fantasy production and, therefore, showed very little aggression in their overt behavior.

In current projective test theory then, the interplay of control and impulse of the subject is quite crucial. In addition, advances in theory make it clear that the subject may be responding not only to the interplay of these two factors but the test instructions, the personality of the examiner, the situation of the testing, and, of course, the material itself. This multiple consideration of the subject's response has broadened a horizon in projective theory and has broadened our base of study of personality theory through projective techniques.

In current developments, projective techniques have been put to the service of evaluating established theories. Dr. Roy Schaefer of Yale University has shown considerable ingenuity in studying Freudian psychoanalytic theory with projective techniques. By applying the Freudian theory of unconscious motivation and intrapsychic conflict, Dr. Schaefer has been able to re- 
fine the use of projective techniques both in clinical work and in research. On the other hand projective theory has been put to the service of personality theory. Dr. Zigmunt Piotrowski in his theory of alphaschizophrenia has been able to demonstrate how theory development can be enriched by projective technique procedures. Piotrowski postulates an alphaschizophrenia in which the control over impulses is greater than the energy output. In this instance, the projective technique findings will give indications of severely distorted personality integration but clinical observation will not show manifest signs of pathology. Projective techniques theory in this instance helps us to understand this apparent discrepancy. A careful scrutiny of the Rorschach record of such subjects indicates that the energy output either is very low and the control is at least moderate or the energy output is moderate and the control is very high. In both these instances the subject maintains apparent personality integration at considerable personal expense. In both Schaefer's research and Piotrowski's research the mutual enrichment of personality theory and projective technique theory is demonstrated and illustrates an important direction of interest in projective tests.

Another important development in projective techniques practice is the development of scales to study limited and circumscribed variables so that a specific personality variable such as aggression or dependency can be studied in depth. Although the isolation of a single variable can do considerable disservice to the study of the complex interacting person, this procedure frequently has considerable merit in developing personality research. A particularly noteworthy procedure has been developed by Dr. Louis Guttman. The method uses a series of graduated stimuli, each with an increasing "pull-power" in eliciting the personality variable. In this mcthod an intensity level can be obtaincd. For example, if one wished to study the level of sexual provocation of an individual, a series of increasingly provocative sexual pictures could be shown the subject for his response. The first of these series might be a picture of a man and a woman sitting in separate seats in a railroad train. The subject, who is particularly sexually responsive might make up a story which would suggest some sexual liaison between the two would soon occur. The person less easily provoked sexually may simply see the two people as passengers in a common transportation carrier. The series could increase in provocativeness so that another picture may show a man and woman close to each other on a park bench with the moon shining overhead. The subject whose sexual provocation level was reached by such a picture might produce a story which would suggest a highly sexualized theme; whereas the subject who was less easily provoked or resisted provocation might see the people in the picture as two students discussing some abstract philosophy and call the moon the sun so as to limit the provoking qualities of the picture. In such a manner, a series of pictures with increasing provocation for the particular personality variable under consideration would be developed.

Although, as in the above illustration many other personality characteristics enter into the subject's perceptual responses beyond that which is the focus of study, attitudes such as defensiveness, selective inattention and concern about the examiner's attitudes toward a story with sexual content, or compensations for loneliness, social unacceptability or for heterosexual intolerance would all influence the subject's responses. The development of instruments, however, to study circumscribed needs or other personality variables is extending the usefulness of projective techniques in personality research even if more and more refinement is necessary. 
Another example of a specific technique for a specific purpose is the Derner Psychosomatic Attitude Pictures which were developed to study the attitudes of tuberculous patients to their illness. In this technique, a pair of pictures for males and a pair for females were drawn. Each pair shows a person in bed and a person standing on a street corner. Without exception the tuberculous patients saw the person in bed as ill with tuberculosis whereas only half of the normal subjects saw the person as ill at all and if ill it was with some inconsequential illness. The picture which showed a person on the street corner was in no case seen as an ill person by the normal population whereas half of the tuberculous population saw the figure as a person who was ill. Underlying all the stories of the tuberculous patient was a sense of fear, worry, anxiety and depression whereas these themes were less frequent in the normal subjects and in many instances the normal subjects gave themes of happiness. Instruments as in this illustration have limited value in total personality study but they supply an important link between personality study and projective techniques.

Along with the development of scaled instruments and procedures for studying specific problems have gone the refinements of the older techniques for better research usefulness. As an example, my student, Dr. Jean W. Sayers, now of Yale University, developed an elaborately refined scoring system for the Rorschach so that the detailed information which the clinician. would ordinarily use in making a clinical judgment would not be lost as in a more gross scoring system. For example, whole responses (W) on the Rorschach were scored so as to differentiate between graduations of vague and precise percepts. Although an elaborate scoring system. may be cumbersome for day to day clinical practice, it tends to follow in a more precise way the many subtle adjustments made by the clinician in his clinical practice. As another example, another of my students, Dr. Anne Lebowitz used a refined Rorschach system which has been. developed on the basis of Dr. Heinz Werner's theory of hierarchization and integration. The particular scoring method used was developed by a number of Dr. Werner's students and appears in its most comprehensive form in a report by Dr. Wesley C. Becker. Dr. Lebowitz, in applying this system of scoring, was able to demonstrate a hierarchy of integration of Rorschach responses for normal subjects, duodenal ulcer subjects and schizophrenic subjects. The research. findings support the theoretical formulations about the differences in personality integration of the three groups and add support to the theory proposed by Dr. Werner.

Another important new frontier of personality research using projective techniques has become possible with the development of research procedures which can deal with multiple data simultaneously. Particularly important is the Q-sort method which allows for the sorting of a number of personality statements in such a manner that at any time if any one statement is moved it affects all the others. This procedure, therefore, makes available an easily manipulative method of simultaneously dealing with many personality descriptive statements in a holistic rather than an atomistic fashion. The method is further valuable since the findings are readily converted into a matrix for factor analytic study. Although the merits of factor analysis are a matter of some dispute, the possibility of applying factor analytic procedures to projective techniques data gives us another means of exploration into personality using projective techniques.

Further research and statistical refinements are necessary and a current trend in the United States continues in this dircction. Particularly additional methods of dealing simultaneously 
with multiple personality characteristics are needed. A frequent complaint by projective techniques theorists is that much of the projective techniques research has been based upon molecular units of personality traits rather than personality configurations. As newer research methods are developed, as better statistical procedures become available, as projective technique theory, personality theory and general psychological theory become refined, we shall be better able to deal with larger groups of personality variables simultaneously. It is interesting to note that whereas the Rorschach research which has used single determinants as a basis for studying personality characteristics has been rather universally negative, the research using the Rorschach in more holistic approaches has tended to have positive findings.

Projective test theory is also expanding to include interpersonal aspects as well as the intrapsychic significance of the responses. A particularly striking example of the significance of interpersonal factors in personality research is shown in a study by Dr. William Haase. Dr. Haase's study illustrates the growing interest in the personality of the experimenter as well as of the subject. He had clinicians rate Rorschachs obtained from subjects who were labelled as of middle class status and similar Rorschachs which were labelled as coming from subjects of lower class status. The clinicians rated those subjects of middle class status as more healthy and frequently, if pathology was believed to be present at all, it was more often diagnosed as a characterological or neurotic difficulty. For those subjects who were purported to be of lower class, the almost similar Rorschachs were rated as less healthy and pathology was frequently diagnosed. Further the diagnoses were of more severe pathology including psychosis. It is important to note that these records were essentially similar but the interpersonal social factor of class difference was important in the interpretation. So as in this illustration, the interaction of the examiner and the subject is becoming increasingly important as an area of projective testing research, possibly growing out of the great interest and practice of psychotherapy by psychologists in the United States. In psychotherapy practice the patient and therapist interactions and personality of both is important.

It may be of interest in our review of the current status and some of the recent directions of projective techniques theory and research to look at the position the techniques hold in present day American psychology. Their status with United States clinical psychologists can be most sharply highlighted by noting that all of the universities in the United States, which teach clinical psychology require their students to take courses and have clinical experience with projective techniques. In the job descriptions for psychologists in mental hygiene clinics, mental hospitals, industry, university counseling centers and in private practice the clinical psychologist is expected to be prepared to do psychotherapy, psychological research and psychological diagnosis. In the latter two instances projective techniques are key tools for psychologists. The techniques most frequently used in the United States include the Rorschach, which is the leading projective technique. Other techniques used are the Thematic Apperception Test and other picture story variations. Many of these picture story techniques are designed for specific purpose such as the series of pictures prepared by Dr. Percival M. Symond which were designed for studying adolescent fantasies. The Children's Apperception Test developed by Dr. Leopold Bellak is designed for studying children. It uses animal figures on the basis that children have highly anthropomorphic notions of animals and therefore find them a ready stimulus for pro- 
jective perceptions. A widely used picture story test for school children is the Michigan Picture Test which includes scenes of family situations, a peer group situation, a child alone, a child with the authority figures of the parent or other persons, a child in the classroom, and a child in stressful situations. Among other projective techniques widely taught and used for diagnostic and research purposes are included figure drawings in which the subject is asked to draw a person. The basic theoretical concept for the Draw-a-person technique is that the figure drawn reflects the person's own body image. Sentence Completion tasks in which a stem of a sentence is given are widely used. These stems for example are such as "I like...." with a blank for the person to fill in or "My mother is...." The Sentence Completion Test allows the person to present to the examiner fairly direct statements about significant topics. Specific stems are developed for specific purposes either for diagnosis or research or published stems are used. Even tests which were originally designed primarily as objective procedures such as the Minnesota Multiphasic Personality Inventory which consists of a series of sentences to which the person replies as to whether it is true, false, or he cannot say about himself has been adapted for projective techniques use.

Some of the projective techniques such as the Pyramid Test which are apparently commonly used in some other countries are rarely used in the United States. The Szondi Test which for a period of time commanded considerable interest in the United States now evokes only limited interest. The change in status of the Szondi is directly related to research with it. A number of researches with the instrument have been essentially negative in their findings. As an example, in the cxcellent study by Dr. Henry P. David it was not possible to differentiate between the epileptics and homosexuals on the basis of the Szondi scores of known epileptics and homosexuals. Not only have research findings been essentially and rather consistently negative, but a further reservation developed from Szondi's theoretical presentation. United States psychologists did not accept the genetic theory which Dr. Szondi proposed as an explanation for the subject's selection of particular pictures. So because of this combination of the research findings being essentially negative, and the theory of doubtful import, the test has had only limited use in the United States.

Handwriting analysis as a projective technique is rarely used by United States psychologists. Unfortunately, graphology is primarily practiced by pseudo-scientists who use superficial check lists to describe personality. Frequently tabloid newspapers carry superficial personality interpretations based on handwriting as an entertainment. The taint of such use of the procedure, along with limited research on the method, has seriously limited it being used by psychologists. It may well be in the futurc this excellent example of the personal style of a person may be more seriously studied by United States psychologists but at this point its usefulness is still to be demonstrated to most of us.

Books on projective techniques continue to pour forth. Among the outstanding publications of recent years is the excellent two volumes by Dr. Bruno Klopfer and co-authors, Developments in Rorschach Technique. The broader coverage of topics in these books beyond the direct clinical technical use is another reflection of the broader interest reported throughout the present paper.

Typically then, American psychologists have adopted projective techniques and projective 
technique theory as a basis for research as well as for clinical application. The major trends in the current status of projective psychology in the United States can be summarized as follows:

1. Projective theory is expanding to include the interpersonal aspects as well as the perceptual and the intrapsychic significance of the response. The study of intrapsychic components has been refined as in the theory of interaction of impulse and control.

2. There is a comprehensive relating of perception and personality theories to projective techniques. As a result projective techniques are being used to measure the dependent variable in psychological research of both a clinical and non-clinical nature.

3. New techniques and techniques with emphasis on a circumscribed variable continue to appear. There are continuing published reports of new techniques which are developed by ingenious psychologists, although most of the new techniques enjoy only a limited adoption.

4. There is continued research activity in the clinical use of projective techniques and the evaluation and refinement of the older projective techniques.

5. Projective techniques as clinical instruments and projective techniques theory has become an accepted part of modern clinical psychology in the United States. Although there is a strong emphasis on the practice of psychotherapy among clinical psychologists they nonethe-less extensively study and use projective techniques as part of their clinical and research functions. Of the published literature on projective techniques in the United States, which is reviewed, the impression could be obtained that there is only linited clinical interest in projective techniques. We tend to overemphasize the research use of the techniques in our publications. In day to day clinical practice, however, they are widely used and their extensive use in research has added to their value, to personality theory and to the better understanding of man.

MS. received I 30,61 .

Gordon F. Derner (1915-) $\quad$ PhD 1950 Columbia Univ. 1951- Assoc, Prof. to Prof., Adelphi College. 